\title{
Lower gastrointestinal bleeding caused by stercoral ulcer
}

\author{
Cheng-Chiang Huang MD, I-Fu Wang MD, Hsin-Hui Chiu MD
}

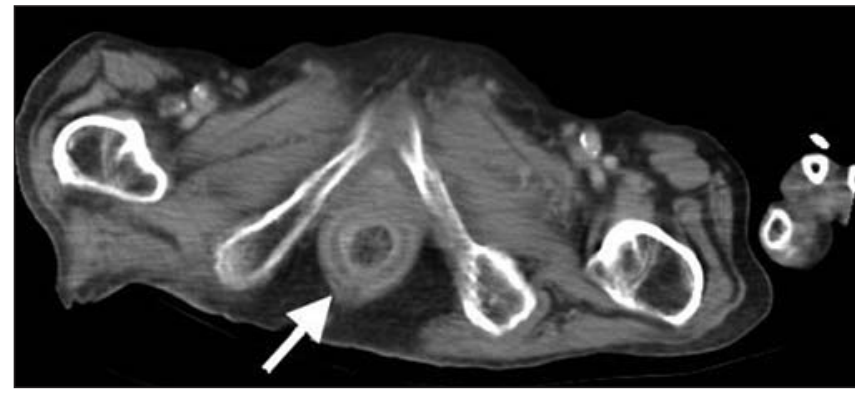

Figure 1: Computed tomographic scan of the abdomen of a man with bloody stool showing a thickened rectal wall (arrow).

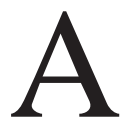

n 85-year-old man with a history of stroke presented with a three-day history of bloody stool. He had chronic constipation treated with herbal medicine, but the constipation had become worse over the past two months. On physical examination, there was marked tenderness of the lower abdomen and right hemiparesis. The patient's hemoglobin level was reduced at $6.39 \mathrm{~g} / \mathrm{L}$. A plain abdominal radiograph showed large amounts of feces in the colon and rectum. Computed tomographic scans of the abdomen showed thickening of the rectal wall (Figure 1) and feces in the colon and rectum.

A colonoscopy following gentle rectal irrigation showed several hard fecalomas inside the ascending colon and transverse colon, and a large, irregular ulcer with active bleeding from a visible vessel within the ulcer base in the rectum (Figure 2). Endoscopic hemostasis with epinephrine injection was successfully performed. Biopsy specimens taken from the ulcer showed chronic and acute inflammation. The patient was discharged on the twelfth day after admission. One month later, a follow-up sigmoidoscopy demonstrated a normal rectum without recurrent bleeding.

Stercoral ulceration, first described in $1894,{ }^{1}$ is thought to be secondary to inspissated or impacted feces causing pressure necrosis of the bowel wall. It usually occurs in elderly patients with a history of chronic constipation. The prevalence of stercoral ulceration is unknown. In autopsy studies, stercoral ulceration has been found in $1.3 \%-5.7 \%$ of elderly patients in long-

From the Departments of Radiology (Huang), Family Medicine (Wang) and Internal Medicine (Chiu), Kuo General Hospital, Tainan, Taiwan

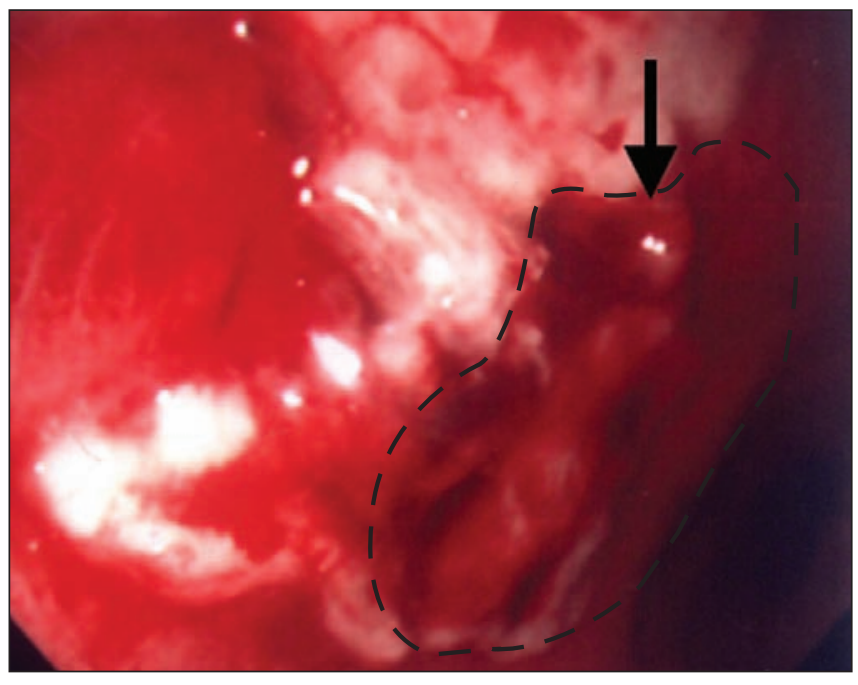

Figure 2: Endoscopic image showing a large, irregular ulcer (outlined) with a bleeding visible vessel (arrow) in the rectum.

term care facilities. ${ }^{2}$ The lesions commonly occur in the rectum and sigmoid colon along the antimesenteric margin. This pattern is thought to be caused by harder consistency of the stool, relatively poor blood supply, a narrow diameter and high intraluminal pressure in this location. Complications of stercoral ulceration include bleeding and perforation. The diagnosis of nonperforating stercoral ulceration is usually based on the endoscopic finding of an irregular, geographically outlined ulcer that conforms to the contour of the impacted feces. Bleeding from stercoral ulcers has been successfully treated with endoscopic hemostasis, including endoscopic multipolar electrocoagulation and injection therapy. ${ }^{3,4}$ Surgical intervention is indicated if stercoral perforation or failure to control bleeding is encountered.

This article has been peer reviewed.

Competing interests: None declared.

\section{REFERENCES}

1. Berry J. Dilatation and rupture of the sigmoid flexure. BMJ 1894;1:301.

2. Madan P, Bhayana S, Chandra P, et al. Lower gastrointestinal bleeding: associated with Sevelamer use. World J Gastroenterol 2008;14:2615-6.

3. Knigge KL, Katon RM. Massive hematochezia from a visible vessel within a stercoral ulcer: effective endoscopic therapy. Gastrointest Endosc 1997;46:369-70.

4. Matsushita M, Hajiro K, Takakuwa H, et al. Bleeding stercoral ulcer with visible vessels: effective endoscopic injection therapy without electrocoagulation. Gastrointest Endosc 1998;48:559 